\title{
MICROFILTRACIÓN EN CAVIDADES CLASE II RESTAURADAS EMPLEANDO TRES ADHESIVOS DE AUTOGRABADO Y DOS MONOFRASCO
}

${ }^{1}$ Lucy Margarita Reyes S., ${ }^{1}$ Sandra Liliana Acevedo A., ${ }^{1}$ Carolina Casella S., ${ }^{2}$ Juan Norberto Calvo R.

${ }^{1}$ Odontóloga, Residente II año Postgrado de Rehabilitación Oral U. Nacional de Colombia.

${ }^{2}$ Odontólogo, Especialista en Rehabilitación Oral, Coordinador del grupo GRIMAD, U. Nacional de Colombia

Autor responsable de correspondencia. Dra. Lucy Margarita Reyes $S$.

Correo electrónico:mare339@hotmail.com

Mención de Honor categoría Posgrados en el Área de Rehabilitación Oral

XIV Encuentro Nacional y III Latinoamericano de Investigación Odontológica. Medellín, Septiembre 18-20 de 2003 Reconocimiento de la Facultad de Odontología de la Universidad Nacional de Colombia. Bogotá, Octubre 3 de 2003

\section{RESUMEN}

Objetivo: El propósito de este estudio fue comparar la microfiltración inmediata después de 1000 ciclos térmicos, en restauraciones clase II utilizando tres adhesivos de auto grabado y dos monofrasco.

Materiales y métodos: Se realizaron cavidades oclusomesiales en ciento veinte premolares extraídos por razones ortodónticas. Cien dientes fueron divididos en cinco grupos de veinte dientes y restaurados con adhesivos: S.E. Clearfil (Kuraray), One Up (Tokuyama), Adper Prompt (3M-ESPE), Excite (Vivadent), Single Bond (3M-ESPE). En los veinte dientes restantes se determinó el área promedio de las cavidades. Diez dientes de cada grupo fueron termociclados durante mil ciclos térmicos, con una temperatura mínima de $6^{\circ} \mathrm{C} \pm 2$ y máxima de $60^{\circ} \pm 2$ en azul de metileno al $0.5 \%$. Las cavidades restauradas fueron cortadas en un microtomo ISOMET en tres planos, obteniendo ocho partes, que fueron analizadas al estereomicroscopio en todas las interfases de unión. La sumatoria de área de microfiltración de cada una de las paredes determinó la microfiltración total. El análisis estadístico fue realizado con ANOVA y el Test de Bartlett's.

Resultados: En todas las muestras hubo microfiltración. El área de microfiltración fue significativamente mayor para los adhesivos de autograbado que para los monofrasco, siendo menor en los grupos no termociclados $(\mathrm{p}<0.001)$.

Conclusiones: La técnica de tres cortes espaciales y tinte, es un método más preciso en el cálculo de la microfiltración. La mayor microfiltración fue observada en los dientes restaurados con Adper Prompt (3M-ESPE), 41.93\%, tanto para los dientes termociclados como para los no termociclados. La menor microfiltración fue observada en los restaurados con Single Bond (3MESPE), 16.47\%. La cara con mayor microfiltración fue la gingival. [Reyes LM, Acevedo SL, Casella C, Calvo JN. Microfiltración en cavidades clase II restauradas empleando tres adhesivos de autograbado y dos monofrasco. Ustasalud Odontología 2004; 3: 15 - 23]

Palabras clave: Microfiltración, Autograbado, Cavidades clase II

\section{MICROLEAKAGE IN CLASS II PREPARATION USING THREE SELF-ETCHING AND TWO SINGLE BOTTLE ADHESIVE SYSTEMS}

\begin{abstract}
objective: This study compared microleakage in class II (Black) preparation using three self-etching and two single bottle adhesive systems.

Materials and methods: One hundred and twenty human extracted premolars due to orthodontic treatment were prepared with a class II (oclusal - medial) preparation. One hundred were restored divided in five groups: 20 with S.E Clearfil (Kuraray), 20 with One Up (Tokuyama), 20 with Adapter Prompt (3M-ESPE), 20 with Excite (Vivadent) and 20 with Single Bond (3M-ESPE). The last twenty were used to determinate the area of the cavity. Ten of each group were term cycled during 1000 cycles, with a low temperature of $6^{\circ} \mathrm{C} \pm 2$ and high of $60^{\circ} \pm 2$.The cavities were restored and cutted with ISOMET of each one eight parts were obtainer and were analyzed at stereomicroscope. The sumatory of microleakage of the parts determined the total microleakage. ANOVA and Bartlett's test were used.

Results: All the samples showed microleakage. Showing the highest the one restored with Adapter Prompt (3M-ESPE), for all the termocycled and not termocycled, and the lowest the ones restored with Single Bond (3M-ESPE). The surface showing the highest microleakage was gingival.

Conclusions: All the self-etching adhesives showed microleakage. All the self-etching adhesives allowed highest microleakage that the ones with single bottle.
\end{abstract}

Key words: Microleakage, Self - etching, Class II preparations

Recibido para publicación: 18 de febrero de 2004. Aceptado para publicación: 30 de mayo de 2004. 


\section{INTRODUCCIÓN}

A través del tiempo, los adhesivos dentinales han evolucionado su composición química y la forma de tratar el sustrato para lograr una mejor adhesión al esmalte y la dentina, buscando disminuir los problemas de microfiltración, sensibilidad y caries recurrente. ${ }^{1,2}$

Los adhesivos de autograbado son materiales dentales recién desarrollados; buscan simplificar la técnica operatoria, disminuir el tiempo de trabajo, conservar una mayor cantidad de tejido orgánico y ser menos agresivos con el órgano dentinopulpar. No eliminan el smear layer, se infiltran a través del mismo y producen un acondicionamiento de la dentina. ${ }^{2}$

El smear layer contiene fibras de colágeno cortado y cristales de hidroxiapatita y por su inherente debilidad puede interferir con una buena adhesión, observándose posibles fallas entre esta capa y la resina curada o fracturas interfasiales entre la dentina intacta mineralizada y el smear layer. ${ }^{3}$ También se han observado fuerzas tensiles con adhesivos de autograbado entre los 10 y $28.5 \mathrm{Mpa}$.

Teóricamente este es un sistema adhesivo, que simultáneamente desmineraliza la dentina y la infiltra, polimerizándose in situ..$^{2-5}$ Con los adhesivos de autograbado se produce la infiltración con un monómero ácido que incrementa su concentración a través del smear layer, en la matriz de dentina inferior, creando una capa híbrida especial denominada pseudo capa híbrida, que contiene una zona superior de smear layer híbrido y una zona inferior de dentina desmineralizada donde penetra el imprimador $(0.5 \mu \mathrm{m})$. Los ácidos anteriores no podían penetrar el smear layer; actualmente los nuevos imprimadores de autograbado tienen ácidos adicionales como el maléico o el nítritico para incrementar la acidez.

De esta forma, aunque la acidez del imprimador puede también ser neutralizada (basificada) por los componentes minerales del smear layer, se puede extender el potencial de penetración del imprimador dentro de la dentina sana, que está debajo.

Esto puede deberse a la presencia física del smear layer como una barrera o su habilidad de basificar la acidez del monómero, haciendo el pH más alto para desmineralizar la dentina inferior intertubular. ${ }^{6}$
En generaciones anteriores los agentes adhesivos tenían una adaptación marginal inadecuada y formación de gaps pos-operatorios; esto se atribuía a la contracción de polimerización de las resinas. ${ }^{7}$ Este problema es todavía observado, aun utilizando adhesivos de esta generación.

Pilo y Ben-Amar observaron que los adhesivos de autograbado presentan un mejor sellado marginal en cemento, pero no muy alto en esmalte comparados con Single Bond y SBMP (3M), los cuales mostraron los mejores resultados sellando el esmalte. ${ }^{8}$

La cantidad y durabilidad del selle marginal en la dentina depende del sistema adhesivo que elija el operador. Alani y Toh, en 1997, demostraron que los factores químicos tóxicos, tales como el ácido y los componentes de los materiales restaurativos por si mismos, tienen una menor significancia como agentes causales del daño pulpar, que la filtración de bacterias alrededor de los márgenes de la restauración. ${ }^{9}$

Existen diferentes maneras de cuantificar la microfiltración; así los tintes, los marcadores químicos, fluorescencia e infiltración de bacterias entre otras. Según Taylor y Lynch (1992), ninguno de estos métodos es ciento por ciento confiable porque la interpretación de los resultados se hace de manera bidimensional y nunca tridimensional..$^{10}$ Además, tradicionalmente los resultados de la microfiltración se dan con indicadores ordinales, nunca con cuantificación del área de microfiltración.

Los objetivos de este trabajo de investigación fueron establecer la eficiencia de la técnica de tres cortes en cada plano espacial y tinte, para determinar el área de microfiltración. Cuantificar y comparar la microfiltración generada al emplear tres adhesivos de Autograbado y dos monofrasco en cavidades clase II y evaluar el efecto del termociclado.

\section{MATERIALES Y MÉTODOS}

Este estudio de tipo cuantitativo experimental se realizó con ciento veinte premolares extraídos por indicaciones ortodónticas, libres de caries y de cualquier tipo de restauración, con cierre apical completo y sin defectos en el esmalte, donados por los pacientes, bajo consentimiento informado. 
Se determinó el área de microfiltración (Variable independiente medida en una escala de razón y proporción) que fue definida como la sumatoria de los valores de filtración del azul de metileno en la interfase de la unión de toda la cavidad, dada en milímetros cuadrados.

Para determinar esta área se tuvieron en cuenta los procesos de termociclado (Variable dependiente de tipo nominal) y los diferentes adhesivos utilizados (Variable dependiente de tipo nominal)

En el presente trabajo diez dientes de cada grupo de estudio fueron termociclados durante 1000 ciclos térmicos, siendo la temperatura mínima $6^{\circ} \mathrm{C} \pm 2$ y la máxima $60^{\circ} \pm 2$.

Los adhesivos utilizados fueron S.E. Clearfil (Kuraray), Adper Prompt (3M-ESPE), One Up (Tokuyama), Excite (Vivadent), Single Bond (3M-ESPE) (Tabla1).

\section{Procedimiento:}

Luego de extraídos los dientes, la muestra fue almacenada en cloramina al $0.5 \%$, refrigerada a $2^{\circ} \mathrm{C}$, hasta el momento del montaje de la prueba. ${ }^{11}$ El período de almacenamiento de los dientes no fue mayor a seis meses.

Setenta y dos horas antes de la realización de las cavidades, los dientes se lavaron y depositaron en agua destilada. Los ciento veinte dientes se midieron en sentido mesodistal, vestíbulolingual o vestíbulopalatino y gingivooclusal.

Luego de realizadas las mediciones, la muestra se dividió al azar en seis grupos de veinte dientes cada uno. Estos se marcaron en la cara vestibular y distal de la corona de acuerdo con el grupo y número de espécimen.
En todos los dientes se realizaron cavidades clase II, en mesial, a dos milímetros de la unión amelocementaria, empleando el estandarizador de cavidades ESCUN, que permitió estandarizar las cavidades (Figura 1).

Luego de realizadas las cavidades en cada grupo de dientes, se le asignó un adhesivo y un material restaurador (Tabla 2).

Todos los adhesivos se aplicaron siguiendo las instrucciones de las casas fabricantes, excepto Excite de Vivadent, pues no se aplicó previo grabado con ácido fosfórico al $37 \%$ sino que se usó como adhesivo de autograbado, para probar el efecto acondicionador por un $\mathrm{pH}$ ácido de 1 , fundamento de la acción autograbable en el sustrato dentario.

\begin{tabular}{cccc}
\hline $\begin{array}{c}\text { Grupo } \\
(\boldsymbol{n}=\mathbf{2 0})\end{array}$ & Adhesivo & Resina & $\begin{array}{c}\text { Casa } \\
\text { Comercial }\end{array}$ \\
\hline 1 & $\begin{array}{c}\text { S.E } \\
\text { Clearfil }\end{array}$ & Clearfil APX & Kuraray \\
2 & $\begin{array}{c}\text { Adper } \\
\text { Prompt }\end{array}$ & P 60 & 3M-ESPE \\
3 & One up & Clearfil APX & Tokuyama \\
4 & Excite & $\begin{array}{c}\text { Tetric } \\
\text { Ceram }\end{array}$ & Vivadent \\
5 & $\begin{array}{c}\text { Single } \\
\text { Bond }\end{array}$ & P60 & 3M-ESPE \\
\hline
\end{tabular}

Tabla 2. Distribución de grupos de acuerdo a los materiales empleados

\begin{tabular}{|c|c|c|c|}
\hline Nombre & $\begin{array}{c}\text { Casa } \\
\text { Comercial }\end{array}$ & $\begin{array}{c}\text { Tipo de } \\
\text { Adhesivo }\end{array}$ & Composición \\
\hline S.E Clearfil & Kuraray & Autograbado & $\begin{array}{l}\text { Acido citrico, } \mathrm{CaCl} 2, \mathrm{NMSA}, 10-\mathrm{MPD} \text {, composite } \\
\text { de microrrelleno }\end{array}$ \\
\hline $\begin{array}{l}\text { Adper } \\
\text { Prompt }\end{array}$ & 3M-ESPE & Autograbado & $\begin{array}{l}\text { Agua, ácido fosfórico, estabilizador, complejos de } \\
\text { fluoruro, parabenos }\end{array}$ \\
\hline One Up & Tokuyama & Autograbado & $\begin{array}{l}\text { Primer, agua, MDP, HEMA, dimetacrilato } \\
\text { hidrofilico, } \\
\text { toluidina }\end{array}$ \\
\hline Excite & Vivadent & Monofrasco & $\begin{array}{l}\text { Monómero de dimetacrilato y acetona, } \\
\text { monómero fosfonato, HEMA, agentes de unión, } \\
\text { silica } 25 \% \text { de etanol }\end{array}$ \\
\hline Single Bond & 3M-ESPE & Monofrasco & Bis-GMA, HEMA, PMDM, fluor, iniciador. \\
\hline
\end{tabular}

Tabla 1. Materiales empleados: Tipo y composición. 
Para la obturación se colocaron los dientes en un modelo simulador de posición (Figura 2) y se restauraron con la resina respectiva, mediante la técnica incremental oblicua, polimerizándose con una lámpara LX3000 de 3M, de $600 \mathrm{mw} / \mathrm{cm}^{2}$ de intensidad de luz, durante el tiempo sugerido por la casa fabricante desde vestibular, lingual o palatino y oclusal, estandarizando esta última distancia a $7 \mathrm{~mm}$ de la pared gingival.

A los 12 minutos de obturados todos los dientes se cubrieron con tres capas sucesivas de barniz de uñas, respetando la periferia de la unión de la restauración hasta un milímetro de la misma.
Todos los dientes fueron almacenados en azul de metileno al $0,5 \%$ y mantenidos en el Higrobath a $37^{\circ} \mathrm{C}$ durante 24 horas.

Los grupos sin termociclado fueron cortados seguidamente y los grupos por termociclar en azul de metileno al $0.5 \%$ a temperaturas de $6^{\circ} \mathrm{C} \pm 2$ durante 30 segundos y $60^{\circ} \mathrm{C} \pm 2$ por 30 segundos, fueron igualmente cortados una vez cumplidos 1000 ciclos (Figura 3A y 3B).

Para facilitar los cortes en el ISOMET (Buheler), la porción radicular de cada diente fue incluida en un cubo de acrílico de $10 \mathrm{~mm}$ por $10 \mathrm{~mm}$.

\begin{tabular}{|c|c|c|c|c|c|c|}
\hline \multirow[t]{2}{*}{ ADHESIVO } & \multicolumn{2}{|c|}{ MICROFILTRACIÓN TOTAL. } & \multicolumn{2}{|c|}{ MICROFLTRACAÓN NO TERMOCICL.ADO } & \multicolumn{2}{|c|}{ MUCROFILTRACIÓN TERMOCICLADO } \\
\hline & Porcentaje & Desviación Estandar & Porcentaje & Desviaciōn Estândar & Porcentaje & Desviación Estandar \\
\hline Clearfil & 36.70 & 14.44 & 42.51 & 12.34 & 31.48 & 14.76 \\
\hline Adper & 41.93 & 13.94 & 47.33 & 12.70 & 36.52 & 13.56 \\
\hline One Up & 33.58 & 16.50 & 44.59 & 12.46 & 22.58 & 12.26 \\
\hline Excite & 34.52 & 18.56 & 34.57 & 20.30 & 34.48 & 17.75 \\
\hline Single Bond & 16.47 & 17.14 & 28.25 & 14.39 & 521 & 10.42 \\
\hline
\end{tabular}

Tabla 3. Porcentaje de área de microfiltración para cada adhesivo en los grupos termociclados y no termociclados.

\begin{tabular}{|c|c|c|c|c|c|c|c|c|}
\hline \multirow[t]{2}{*}{ ADHESIVO } & \multicolumn{2}{|c|}{ VESTIBULAR } & \multicolumn{2}{|c|}{ PALATINO LINGUAL } & \multicolumn{2}{|c|}{ AXIAL } & \multicolumn{2}{|c|}{ GINGIVAL } \\
\hline & Porcentaje & $\begin{array}{c}\text { Desviación } \\
\text { Estándar }\end{array}$ & Porcentaje & $\begin{array}{c}\text { Desviación } \\
\text { Estándar }\end{array}$ & Porcentaje & $\begin{array}{c}\text { Desviación } \\
\text { Estándar }\end{array}$ & Porcentaje & $\begin{array}{c}\text { Desviacion } \\
\text { Estindar }\end{array}$ \\
\hline Clearfil & 2.07 & 1.17 & 2.39 & 1.59 & 1.69 & 0.58 & 2.06 & 0.97 \\
\hline Adper & 2.77 & 4.27 & 2.67 & 0.89 & 1.64 & 0.50 & 4.13 & 8.37 \\
\hline One Up & 2.38 & 3.66 & 1.87 & 1.08 & 1.54 & 0.64 & 3.72 & 7.04 \\
\hline Excite & 2.23 & 2.54 & 1.85 & 0.98 & 1.75 & 0.79 & 2.86 & 4.60 \\
\hline Single Bond & 1.55 & 3.83 & 2.64 & 6.51 & 1.05 & 0.87 & 0.84 & 1.17 \\
\hline
\end{tabular}

Tabla 4. Porcentaje de área de microfiltración por caras para dientes termociclados y no termociclados.

\begin{tabular}{|c|c|c|c|c|c|c|c|c|}
\hline \multirow[t]{2}{*}{ ADHESTVO } & \multicolumn{2}{|c|}{ VESTIBULAR } & \multirow{2}{*}{$\begin{array}{c}\text { PALATIN } \\
\text { Porcentaje }\end{array}$} & \multirow{2}{*}{$\begin{array}{l}\text { L.INGUAL } \\
\text { Desviación } \\
\text { estăndar }\end{array}$} & \multicolumn{2}{|c|}{ AXIAL. } & \multicolumn{2}{|c|}{ GINGTVAL. } \\
\hline & Porcentaje & $\begin{array}{c}\text { Desviación } \\
\text { estándar }\end{array}$ & & & Porcentaje & $\begin{array}{c}\text { Desviación } \\
\text { estándar }\end{array}$ & Porcentaje & $\begin{array}{c}\text { Desviación } \\
\text { eståndar }\end{array}$ \\
\hline Clearfil & 1.70 & 1.04 & 1.56 & 0.95 & 1.64 & 0.77 & 1.47 & 1.00 \\
\hline Adper & 2.98 & 5.98 & 2.52 & 0.87 & 1.62 & 0.42 & 5.70 & 11.91 \\
\hline One Up & 1.77 & 3.82 & 1.15 & 0.86 & 1.25 & 0.64 & 3.16 & 7,65 \\
\hline Excite & 2,48 & 3.32 & 1,69 & 0.94 & 1.91 & 0.76 & 4,02 & 6.36 \\
\hline Single Bond & 1,04 & 4.53 & 3.21 & 8.93 & 0,43 & 0.65 & 0.29 & 0.95 \\
\hline
\end{tabular}

Tabla 5. Porcentaje de área de microfiltración por caras para dientes no termociclados.

\begin{tabular}{ccccccccc}
\hline ADHFSTvo & \multicolumn{2}{c}{ VESTIBULAR } & \multicolumn{2}{c}{ PALATINO LINGUAL } & \multicolumn{2}{c}{ AXIAL. } & GINGIVAL \\
\hline & Porcentaje & $\begin{array}{c}\text { Desviación } \\
\text { estándar }\end{array}$ & Porcentaje & $\begin{array}{c}\text { Desviación } \\
\text { estándar }\end{array}$ & Porcentaje & $\begin{array}{c}\text { Desviación } \\
\text { estándar }\end{array}$ & $\begin{array}{c}\text { Porcentaje } \\
\text { Desviación } \\
\text { estándar }\end{array}$ \\
\hline Clearfil & 2.49 & 1.18 & 3.31 & 1.70 & 1.74 & 0.28 & 2.71 & 1.08 \\
Adper & 2.57 & 1.09 & 2.83 & 0.93 & 1.66 & 0.59 & 2.57 & 0.80 \\
One Up & 3.00 & 3.45 & 2.59 & 0.55 & 1.82 & 0.53 & 4.28 & 6.74 \\
Excite & 1.98 & 1.40 & 2.05 & 1.04 & 1.59 & 0.83 & 1.70 & 1.15 \\
Single Bond & 1.55 & 2.83 & 2.02 & 2.21 & 1.73 & 0.48 & 1.44 & 1.14 \\
\hline
\end{tabular}

Tabla 6. Porcentaje de área de microfiltración por caras para dientes termociclados.

18 




Figura 1. Estandarizador de cavidades (ESCUN)

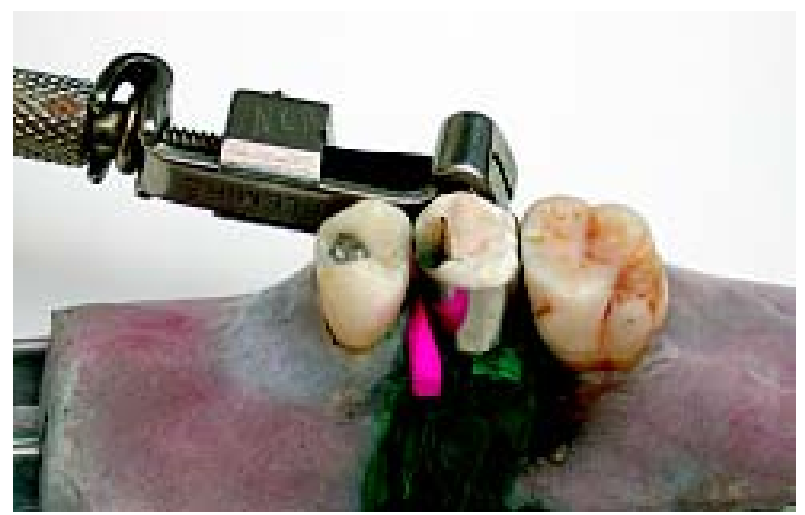

Figura 2. Simulador de posición

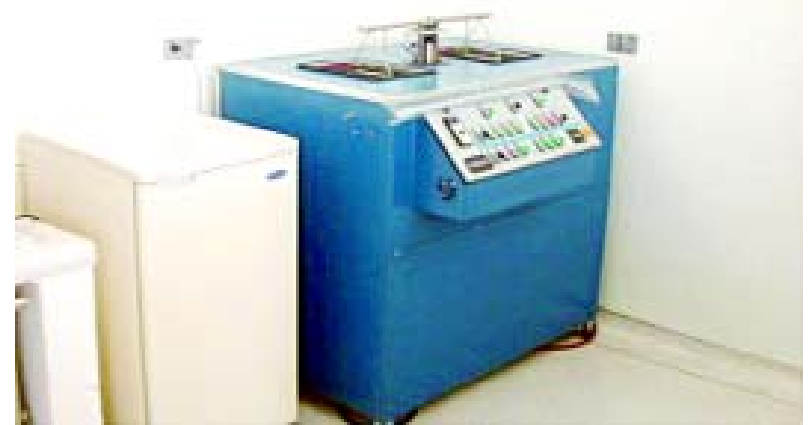

Figura 3A. Termociclador TERMOCYL

Se hicieron los siguientes cortes: Uno en sentido mesodistal incidiendo la restauración en la mitad, un corte transversal a dos milímetros de la pared gingival, y el tercero a 1.5 milímetros de la pared axial de la cavidad (Figuras 4 y 5).

Con estos tres cortes se obtuvieron 8 partes, observadas a 45X por todas sus paredes con el estereomicroscopio LEICA 2000, midiendo en micras la filtración del azul de metileno en la interfase resina- diente. Conocida la filtración en cada interfase, se calculó el área de filtración de las cuatro pare-



Figura 3B. Micrótomo ISOMET



Figura 4. Esquema de los cortes realizados en la restauración y obtención de las 8 partes.

des (gingival, axial, palatino y vestibular) (Figuras 6, 7 y 8). Estas cuatro áreas fueron sumadas para determinar el área $\left(\mathrm{mm}^{2}\right)$ de microfiltración total de la cavidad.

Los veinte dientes que no fueron restaurados, se utilizaron para determinar el área promedio de la cavidad. Para esto se tuvo en cuenta el diámetro de la fresa $(1.4 \mathrm{~mm})$, el ancho del disco del micrótomo $(0.380 \mathrm{~mm})$, la profundidad del surco y las medidas de la cavidad.

Con base en este promedio, considerado el 100\% de área, se calculó el porcentaje de filtración de cada cavidad tomando como valor la media para cada grupo.

\section{Análisis estadístico:}

Los resultados fueron analizados utilizando el análisis de varianza ANOVA en todas las variables, obteniendo las probabilidades, los promedios, la desviación estándar y la frecuencia para cada uno de los datos. 


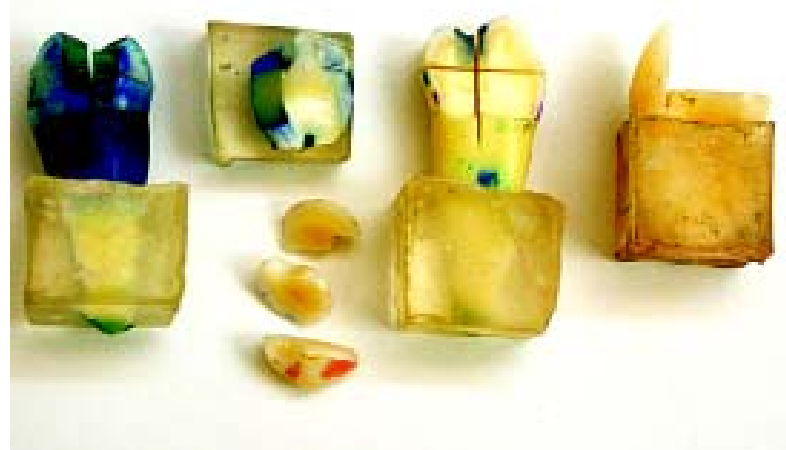

Figura 5. Microcortes obtenidos con el isomet

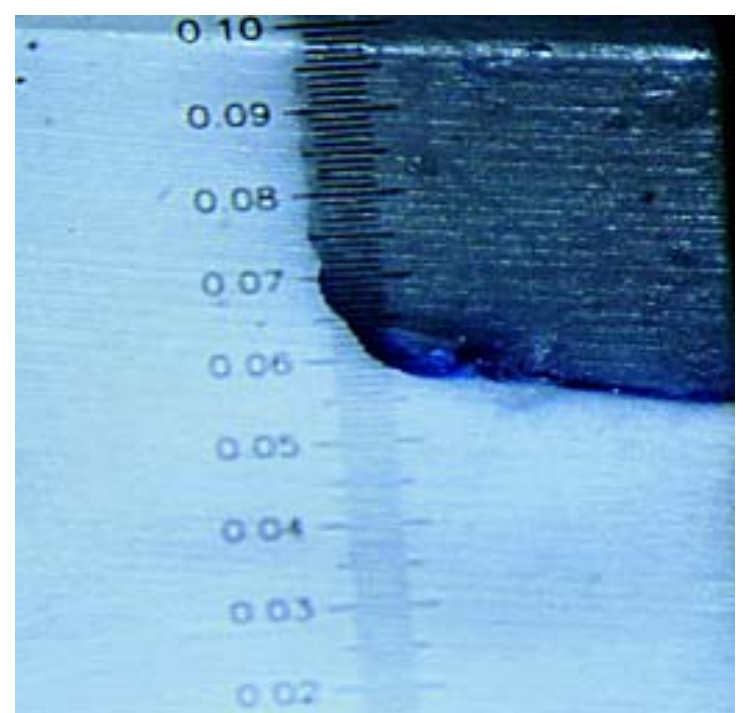

Figura 7. Microfiltración en la pared gingival de la cavidad Clase II restaurada con Adper Prompt - Z 250 (3m-ESPE), en un diente no termociclado.

Además se utilizó el test de Bartlett's para evaluar la homogeneidad de las varianzas. En esta prueba la probabilidad debió ser superior a 0.05 para justificar la aplicación del ANOVA.

\section{RESULTADOS}

Todas las restauraciones en los diferentes grupos, presentaron microfiltración.

El porcentaje promedio de microfiltración en los dientes no termociclados fue 25.65 con una desviación estándar de 17.82. Para los dientes termociclados el porcentaje de microfiltración fue 39.51, con una desviación estándar de 15.79 .

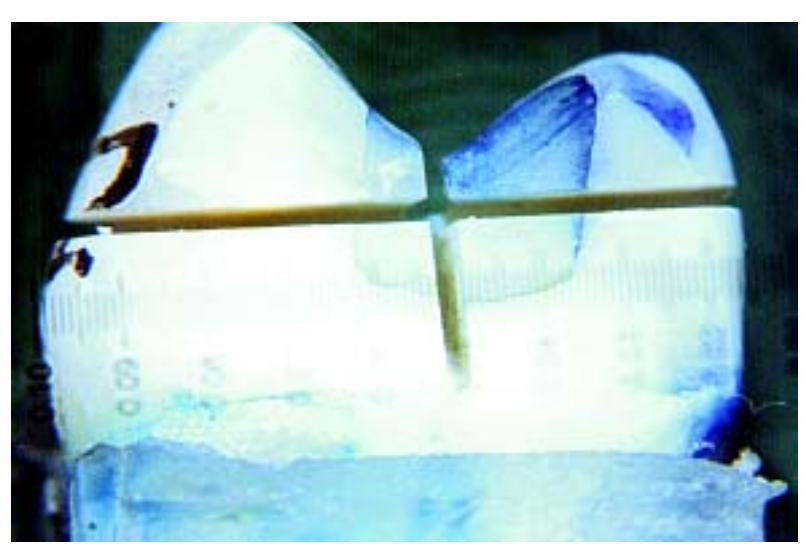

Figura 6. Corte vertical y horizontal de restauración en diente no termociclado.

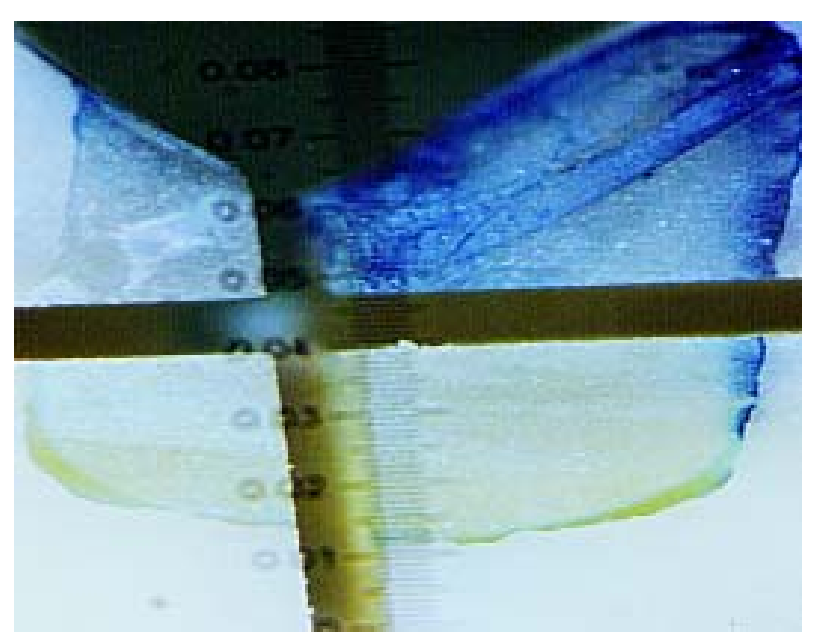

Figura 8. Microfiltración cara vestibular de la cavidad clase II restaurada con Excite-Tetric ceram (Vivadent), en un diente termociclado.

Los porcentajes de microfiltración de los dientes no termociclados (prob $<$ F0.0001, $c^{2}: 0.580$ ) y termociclados (prob $<$ F0.0453, $\mathrm{c}^{2}: 0.488$ ) son estadísticamente significativos.

Grupos no termociclados: El mayor porcentaje de microfiltración de los dientes restaurados no termociclados fue para el grupo con adhesivo Adper Prompt (3M-ESPE) con un 36.52\% y el menor valor fue el de Single Bond (3M-ESPE) con 5.21\% (Tabla 3).

Grupos termociclados: En los dientes termociclados el mayor porcentaje de microfiltración fue también para el grupo con Adper Prompt (3M-ESPE) con un 47.33\% y el menor con Single Bond (3M-ESPE) con un 28.25\% (Tabla 3). 
En el análisis general de la filtración por cada cara y por cada adhesivo se encontró:

Cara gingival: La microfiltración expresada como $\mathrm{mm}^{2}$ en la pared gingival de los dientes no termociclados fue menor en Single Bond $\left(0.29 \mathrm{~mm}^{2}\right)$ y mayor en Adper Prompt $\left(5.70 \mathrm{~mm}^{2}\right)$. En los dientes termociclados también es menor en Single Bond $\left(1.14 \mathrm{~mm}^{2}\right)$ y mayor en One Up $\left(6.74 \mathrm{~mm}^{2}\right)$.

Los valores de filtración obtenidos en el área gingival no son estadísticamente significativos para los dientes no termociclados (prob $<$ F0.4273, $c^{2}: 0.000$ ) y termociclados (prob < F0.3149, C $^{2}: 0.000$ )

Cara vestibular: El área de microfiltración mayor de la cara vestibular de los dientes restaurados no termociclados fue para Adper Prompt $\left(2.98 \mathrm{~mm}^{2}\right)$ y la menor para el Single Bond $\left(1.04 \mathrm{~mm}^{2}\right)$.

La microfiltración vestibular de los dientes restaurados termociclados fue mayor en One Up $\left(3.00 \mathrm{~mm}^{2}\right)$ y menor en Single Bond $\left(2.10 \mathrm{~mm}^{2}\right)$.

El área de microfiltración vestibular de los dientes termociclados (prob < 0.265, c2:0.000) y no termociclados no es estadísticamente significativa (prob $<$ F0.233, $\left.c^{2}: 0.000\right)$.

Cara palatina o lingual: La mayor área de microfiltración en la cara palatina o lingual de los dientes no termociclados fue para single bond (3.21 $\mathrm{mm}^{2}$ ) y la menor fue para Adper Prompt $\left(2.52 \mathrm{~mm}^{2}\right)$.

Sin embargo en los dientes termociclados la menor área fue medida en Single Bond $\left(2.02 \mathrm{~mm}^{2}\right)$ y la mayor en S.E Clearfil $\left(3.31 \mathrm{~mm}^{2}\right)$.

Los valores de microfiltración en la cara palatina 0 lingual, en los dientes termociclados (prob < F0.8061, $\left.c^{2}: 0.001\right)$ y no termociclados (prob $<$ F0.8061, $c^{2}: 0.000$ ) no son estadísticamente significativos.

Cara axial: En la cara axial de los dientes sin termociclar, la mayor microfiltración fue medida con el Excite ( $\left.1.91 \mathrm{~mm}^{2}\right)$ y la menor con el Single Bond (0.43 $\mathrm{mm}^{2}$ ); en los dientes termociclados, la mayor área fue para One Up $\left(1.82 \mathrm{~mm}^{2}\right)$ y la menor para Excite (1.59 $\mathrm{mm}^{2}$ ).
El área de microfiltración gingival en los dientes termociclados (prob < F0.9812, $c^{2}: 0.069$ ) no es estadísticamente significativa; en cambio, en los no termociclados (prob < F0.0001, $\mathrm{c}^{2}: 0.464$ ) si lo es.

Todos los datos netos de área de filtración fueron calculados como porcentaje, basados en el área promedio de las cavidades y paredes (100\% de área); están condensados en las tablas 3-6.

\section{DISCUSIÓN}

Los resultados de este estudio no son comparables cuantitativamente con otros, por existir diferencias metodológicas, de indicadores y criterios de cálculo. Se utilizó un estandarizador de cavidades (ESCUN), que permitió realizar las cavidades con la misma angulación, amplitud, distancia de la unión amelocementaria $(2 \mathrm{~mm})$.

También se utilizó un modelo simulador de posición, que imitó las dificultades clínicas durante la obturación y la polimerización de las restauraciones. Además, se estandarizó la distancia de polimerización a 7 milímetros de la pared gingival en todas las muestras.

La manera de medir la microfiltración fue hecha en tres dimensiones. Taylor y Lynch concluyen en su estudio que existen deficiencias en la interpretación de los resultados de microfiltración cuando es medida por penetración de marcadores en un solo corte, ya que este método permite hacer una interpretación bidimensional y no tridimensional. ${ }^{10}$

Este estudio midió la microfiltración en los tres planos del espacio. Para esto se determinó el área de cada una de las paredes de la cavidad y a partir de esta medición el área total, lográndose determinar el porcentaje de microfiltración del azul de metileno en la interfase de la restauración. No se utilizaron parámetros ordinales de $0 \mathrm{a} 5$, como es reportado por varios trabajos, entre ellos Barbieri, que utilizando penetración de tintes, encontró microfiltración en todas las muestras utilizadas en su estudio; sin embargo, concluyó que ésta era menor cuando se fotopolimerizaba el adhesivo junto con la resina fluída. ${ }^{12}$

Tay, en 1995, utilizando microscopía electrónica de transmisión clasificó la microfiltración como poca, modera- 
da y agresiva, de acuerdo con la capacidad del adhesivo de autograbado para desmineralizar la dentina. ${ }^{7}$ En el 2000 , con adhesivo de autograbado en que variaba el solvente, clasificó la microfiltración en la interfase resina-adhesivo con parámetros de 0 a 5.6

Se ha observado mediante microscopía electrónica de barrido que los adhesivos, previo acondicionamiento, tienen la capacidad de formar una capa híbrida, con un espesor menor en la técnica de autograbado. ${ }^{13}$

La microfiltración es el principal problema en la restauración de cavidades clase II, y está relacionado con sensibilidad postoperatoria, caries recurrente y pigmentación de los bordes de la restauración. 8, 14,15 Miller y colaboradores, en el 2000, encontraron microfiltración en todas las cavidades siendo de difícil control, por la incapacidad de los adhesivos dentinarios ("multipropósito"), para sellar esmalte; por la dificultad de adaptar la resina sobre todo en gingival, sumado a la complejidad en la polimerización. Primero, en esmalte el sellado es de pobre calidad o está ausente, porque debe hacerse con adhesivos dentinarios, que no muestran capacidad de hacerlo.

En nuestro estudio, la pared con mayor microfiltración fue la gingival (0.84 - $\left.4.13 \mathrm{~mm}^{2}\right)$. Perdigao, ${ }^{1,2}$ Reeves, ${ }^{15} \mathrm{y}$ Abate ${ }^{16}$ entre otros, afirman que una posible causa de la gran microfiltración en esta pared se deba, quizas, a su cercanía con la unión amelocementaria, dado que en esta zona los túbulos dentinales tienen un diámetro mayor. Además, el cemento radicular es un tejido con un alto componente orgánico y el selle puede verse afectada por esta razón., ${ }^{2,3}$

Vargas, en 1999, encontró que la capa de colágeno no es importante en el mecanismo de adhesión entre la dentina y el adhesivo, ya que el primero puede inhibir la penetración del material dentro del tejido desmineralizado. Si existe eliminación del colágeno libre, puede existir una mejor difusión del adhesivo dentro de la dentina desmineralizada. ${ }^{17}$

Brakett y colaboradores evaluaron la microfiltración en tres materiales restauradores en preparaciones cavitarias clase $\mathrm{V}$ por vestibular y lingual en incisivos bovinos. Las preparaciones estuvieron centradas en la unión amelocementaria y fueron preparadas con biseles de $45^{\circ}$ en esmalte encontrando que la mayor incidencia de microfiltración está en la pared gingival, especialmente cuando se utilizan adhesivos de autograbado. ${ }^{18}$
En el presente estudio la mayor microfiltración la tuvieron los dientes en los que se utilizaron adhesivos de autograbado. Adper Prompt (3M-ESPE) tiene una microfiltración del $41.93 \%$, S.E. Clearfil (Kuraray) del 36.70\% y One Up (Kuraray) del 33.58\% (Tabla 3).

Estos adhesivos acondicionan el smear layer, lo infiltran y lo involucran en la adhesión. Tradicionalmente esta capa es considerada como un obstáculo para la adhesión, pues es una capa delgada, contaminada, presenta grietas y es degradable, además actúa como buffer, disminuyendo el potencial de grabado del adhesivo sobre la dentina., ${ }^{2,3}$

Perdigao y De Souza Costa afirman que la microfiltración depende del tiempo de degradación de la interfase de la restauración y que la adhesión depende del grosor de la capa híbrida, que es mayor cuando se utiliza la técnica de grabado total. Concluyen que la simplificación del procedimiento, como en los adhesivos de autograbado, no es garantía de éxito en una restauración., ${ }^{2,4}$

Excite (Vivadent) tuvo una microfiltración de 34.52\%. Este adhesivo fue manejado como un adhesivo de autograbado, debido a su pH de 1. De esta manera se refuta la teoría, que afirma que los adhesivos con $\mathrm{pH}$ ácido, pueden grabar por sí mismos el tejido dental y asegurar un perfecto selle marginal. Esto permite pensar que el $\mathrm{pH}$ no es el único factor de éxito, sino también los componentes y las características fisicas del autograbador.

Este adhesivo contiene PENTA, molécula de tipo multifuncional, que puede desmineralizar parcialmente la dentina y facilitar la penetración de los demás componentes del mismo dentro del tejido dentinal; sin embargo, eso no ocurre en el esmalte periférico, requiere un grabado ácido previo, cuando es utilizado sobre este tejido. ${ }^{19,20}$

El porcentaje de microfiltración para el Single Bond (3MESPE) fue $5.21 \%$, sin termociclado y $28.86 \%$ con termociclado. Esto comprueba que el uso del grabado ácido es un aspecto fundamental dentro de los procedimientos de la restauración para favorecer el sellado marginal.

El grabado ácido aumenta la superficie de contacto de los adhesivos, tanto en esmalte como en dentina, y disminuye el porcentaje de filtración dentro de las restauraciones, especialmente en las cavidades clase II, por la ubicación de las mismas dentro de la cavidad oral., ${ }^{2,3}$

Pilo y colaboradores comprobaron que el Single Bond y el 
Scotchbond multipropósito, utilizando la técnica de grabado total, son los adhesivos que tienen un mejor sellado en el margen del esmalte. ${ }^{8}$ Sin embargo, Vargas nego que la capa de colágeno sea importante en el mecanismo de adhesión entre la dentina y el adhesivo, pues a partir de la eliminación del colágeno libre encontraron valores altos de adhesión. ${ }^{17}$

El hecho que los dientes no termociclados (llevados al tinte dentro de las primeras tres horas de restaurados) tuvieran filtración, demuestra que inicialmente no existe un selle completo y adecuado, producido posiblemente por los bajos grados de conversión química de los materiales, por la posible degradación inicial y por la contracción de polimerización.

Al entrar en función, el adhesivo sufre una degradación, porque no existe compatibilidad del material y el medio húmedo que lo rodea, por fatiga térmica del mismo o por un bajo grado de conversión química y favorece la penetración de enzimas bacterianas y otras sustancias tóxicas. ${ }^{6}$

En las condiciones de este estudio se puede concluir:

1. La técnica implementada de tres cortes en cada plano espacial y tinte, es un método complejo, pero de mayor precisión en el cálculo de la microfiltración frente a los análisis bidimensionales.

2. Todas las cavidades, clase II restauradas con resinas compuestas, presentaron filtración independientemente del acondicionamiento.

3. La microfiltración en cavidades clase II restauradas con resinas compuestas fue mayor cuando se utilizaron adhesivos de autograbado.

4. La mayor microfiltración total se produjo usando Adper Prompt (3M-ESPE) y la menor con Single Bond (3M-ESPE)

5. La pared con mayor microfiltración fue la gingival.

\section{BIBLIOGRAFÍA}

1. Swift EJ Jr, Perdigao J, Heymann HO. Bonding to enamel and dentin: A brief story and state or art. Quintessence International 1995; 26: 95 110.

2. Perdigao J. Dentin bonding as a function of dentin structure. Dent Clin North Am 2002; 46: 277 - 301.

3. Koibuchi H. Bonding to dentin with a self-etching primer: The effect of smear layers. Dent Mater 2001; 17: 122 - 126.

4. De Souza Costa CA, Lopes do Nascimento AB, Teixeira HM, Fontana UF. Response of human pulps capped with a self-etching adhesive system. Dent Mater 2001; 17: 230 - 240.
5. Hashimoto M, Ohno H, Endo K, Kaga M, Sano H, Oguchi H. The effect of hybrid layer on bond strength: desmineralized dentin zone of hybrid layer. Dent Mater 2000; 16: 406 - 411.

6. Tay FR, Gwinnet AJ, Pang KM, Wei SH. Variability in microleakage observed in total etch wet-bonding technique under different handling conditions. J Dent Res 1995; 74: 1168 - 1178.

7. Tay FR, Pashley DH. Aggressiveness of contemporary self etching systems. I: Depths of penetration dentin smear layers. Dent Mater 2001; 17: 296 - 308.

8. Pilo R, Ben-Amar A. Comparation of microleakage for three one-bottle and three múltiple step dentin bonding agents. J Prosthet Dent 1999; 82: 209 - 213.

9. Alani AH, Toh CG. Detection of microleakage around dental restorations: a review. Oper Dent 1997; 22: 173 - 185.

10. Taylor MJ, Lynch E. Microleakage. J Dent Res 1992; 20: 3 - 10.

11. Sano H, Yoshiyama M, Ebisu S, Burrow MF, Takatsu T, Ciucchi B, et al. Comparative SEM and TEM observations of nanoleakage within the hybrid layer. Oper Dent 1995; 20: 160 - 167.

12. Bedran de Castro AK, Pimenta LA, Amaral CM, Ambrosano GM. Evaluation of microleakage in cervical margins of various posterior restorative systems. J Esthetic Dentistry 2002; 14: 107 - 114.

13. Masson et al. Preliminary evaluation of the marginal of a compomers.

14. Wendt SL, McInnes PM, Dickinson GL. The effect of thermocycling in microleakage analysis. Dent Mater 1992; 8: $181-184$.

15. Reeves GW, Fitchie JG, Hembree JH Jr, Puckett AD. Microleakage of new dentin bonding systems using human and bovine teeth. Oper Dent 1995; 20: 230 - 235.

16. Abate PF, Bertacchini SM, Polack MA, Macchi RL. Adhesion of a compomer to dental structures. Quintessence International 1997; 28: 509 - 512.

17. Vargas CM. Resin - dentin shear bond strength and interfacial ultrastructure with and without a hybrid layer. Oper Dent 1999; 22: 159 - 166.

18. Brackett WW, Gunnin TD, Gilpatrick RO, Browning WD. Microleakage of class $\mathrm{V}$ compomer and light-cured glass ionomer restorations. JProsthet Dent 1998; 79: $261-263$.

19. Roulette. Adhesion the silent revolution in dentistry. Quintessence Books 2000.

20. McLean JW. The pioneers of enamel and dentin bonding. J Adhes Dent 1999; 1: 185 - 187. 